\title{
Estimulação magnética
}

transcraniana de baixa freqüência no

tratamento da depressão

Low Frequency Transcranial Magnetic Stimulation for Treatment of Depression

RAPHAEL BoEChAT-BARROS ${ }^{1}$

\begin{abstract}
Resumo
Este artigo descreve aspectos neurofisiológicos e clínicos do uso da estimulação magnética transcraniana repetitiva (EMTr), especialmente a de baixa freqüência. Técnicas de neuroimagem e hipóteses sobre o funcionamento da EMTr em longo prazo são abordados. Alguns resultados de estudos que envolvem EMTr de baixa freqüência no tratamento da depressão são citados, especialmente um estudo realizado na Universidade de Brasília usando a aplicação da EMTr de baixa freqüência $(0,5 \mathrm{~Hz})$, duas vezes por semana, durante quatro semanas, em dez pacientes. Neste estudo utilizando a escala de Hamilton de 17 itens, os pacientes foram analisados em três momentos: T-0, T-1 e T-2, respectivamente, início, meio e final das aplicações. Como resultado se observou um decréscimo significativo $(\mathrm{p} \leq 0,01)$ nas suas pontuações, quando comparados os três momentos, utilizando-se o teste $\mathrm{x}^{2}$ de Friedman. As possíveis vantagens desta técnica são discutidas.
\end{abstract}

Palavras-chave: Estimulação magnética transcraniana, baixa freqüência, depressão.

\begin{abstract}
This article describes neurophysiological and clinical aspects of repetitive transcranial magnetic stimulation (rTMS), especially low frequency rTMS. Neuroimaging techniques and hypothesis about long term actions of rTMS are discussed. Some results of low frequency rTMS on the treatment of depression are mentioned, especially one study in the University of Brasilia using low frequency rTMS twice a week, during 4 weeks in 10 patients. In this study, using the 17 item Hamilton scale, patients were scored at three different moments: T- $0, \mathrm{~T}-1$ and T- 2 , respectively, at the beginning, at the middle and at the end of the treatment. According to $x^{2}$ Friedman's test there was a significant decrease $(p \leq 0,01)$ in depression scores. When the three moments were compared. Possible advantages of this technique are discussed.
\end{abstract}

Keywords: Transcranial magnetic stimulation, low frequency, depression.

Recebido: 12/09/2004 - Aceito: 01/10/2004

1 Psiquiatra, doutorando em Ciências da Saúde pela Universidade de Brasília - UnB

Endereço para correspondência: Laboratório de Neurociências e Comportamento, Instituto de Biologia, ICC Sul, Módulo 8, Universidade de Brasília, Asa Norte, 70910-100, Brasília, DF. Fone: (61) 346-1870; e-mail: boechat@unb.br 
A estimulação magnética transcraniana (EMT) pode ser de pulsos únicos ou repetitivos. Em relação ao número de pulsos por unidade de tempo, existem dois tipos de estimulação magnética transcraniana repetitiva (EMTr): baixa freqüência $(\leq 1 \mathrm{~Hz})$ e alta freqüência $(>1 \mathrm{~Hz})$, com efeitos opostos. O uso da estimulação magnética de alta freqüência aumenta o fluxo sangüíneo cerebral na área, com conseqüente aumento da atividade cerebral. A estimulação de baixa freqüência, por outro lado, diminui a atividade cerebral (Speer et al., 2000).

Existem duas possibilidades de aplicação da EMTr no tratamento da depressão. Inicialmente, George et al. (1995) e logo em seguida Pascual-Leone et al. (1996) propuseram utilizar alta freqüência aplicada sobre o córtex pré-frontal dorsolateral esquerdo, com intuito de aumentar a atividade daquela área que estaria hipofuncionante na depressão. Posteriormente, Menkes et al. (1999) sugeriam que a depressão maior deva ser resultado de uma diminuição da função do lobo frontal esquerdo em relação ao direito. Baseados nesta hipótese propuseram o tratamento com a estimulação magnética transcraniana de baixa freqüência sobre o córtex pré-frontal dorsolateral direito, com intuito de diminuição da atividade naquela área.

\section{Técnicas de neuroimagem e EMTr}

Em contraste com o rápido crescimento da literatura sobre os efeitos clínicos da EMTr, ainda são poucos os estudos sobre os mecanismos neurobiológicos envolvidos (Li et al., 2004).

Estudos com indivíduos saudáveis durante ou imediatamente após a aplicação da EMTr, envolvendo ressonância nuclear magnética funcional (fMRI), tomografia por emissão de pósitrons (PET) e tomografia por emissão de fóton único (SPECT), têm demonstrado que ela seria capaz de produzir mudanças na atividade tanto em regiões corticais abaixo da bobina, como em regiões corticais e subcorticais. Assim, os efeitos da EMTr não são apenas na área cortical atingida diretamente pelo campo magnético; uma série de circuitos e conexões cerebrais encarregam-se de levá-lo a áreas distantes no cérebro (Keck, 2003).

O primeiro estudo que envolveu uma mensuração dos efeitos da EMTr, através do PET, foi conduzido por George et al. (1995), sugerindo que, após duas semanas de tratamento diário de EMTr, ocorreria um aumento do metabolismo cerebral em algumas áreas. Teneback et al., em 1999, demonstraram, utilizando o SPECT, que os pacientes deprimidos que responderam ao tratamento de dez dias de EMTr, comparados aos não-respondedores, apresentaram um aumento na atividade no lobo frontal inferior, bem como áreas do sistema límbico.
A maioria dos estudos de neuroimagem que envolvem a aplicação de EMTr em pacientes deprimidos demonstra os resultados após algumas semanas da aplicação, os quais tendem a sugerir uma ativação frontal bilateral em regiões límbicas e paralímbicas (Li X et al., 2004).

Loo et al. (2003), utilizando o SPECT, demonstraram que EMTr a $1 \mathrm{~Hz}$ aplicada sobre o córtex préfrontal leva a um aumento imediato do fluxo sangüíneo cerebral no cíngulo anterior direito, córtex parietal bilateral e ínsula, além do cerebelo esquerdo.

Recentemente, Li et al. (2004), utilizando EMTr de $1 \mathrm{~Hz}$ sobre o córtex pré-frontal dorsolateral esquerdo observaram, através da fMRI, um aumento imediato da atividade no local da aplicação, bem como em conexões límbicas: córtex pré-frontal medial bilateral, córtex frontal orbital direito, hipocampo esquerdo, núcleo mediodorsal do tálamo, putâmen bilateral, ínsula bilateral e giro temporal bilateral. Estas informações sugerem que não apenas o córtex pré-frontal estaria envolvido na resposta terapêutica de pacientes deprimidos à EMTr, mas regiões límbicas, que sabidamente estão relacionadas com a depressão, também podem ter seu funcionamento alterado. Embora a maioria dos estudos sugira uma ativação local no momento da aplicação da EMTr, alguns poucos demonstram resultados opostos; isto pode ser explicado pela diferença nas populações e parâmetros utilizados (Li et al., 2004).

Nadeau et al. (2002), estudando o fluxo sangüíneo através do SPECT em pacientes deprimidos, antes e após aplicação de EMTr, sugerem que existem diferentes padrões de atividade cerebral na depressão, mensuráveis pelo fluxo sangüíneo, bem como diferentes padrões de resposta ao tratamento. Pode existir mais de um estado de depressão, ou a depressão pode estar relacionada com mais de um modelo neurofisiológico, com grande variedade interpessoal de acordo com as vivências pessoais.

\section{EMTr, plasticidade neuronal e efeitos em longo prazo}

Pascual-Leone et al. (1994), aplicando freqüências de 1, $3,5,10,20$ e $25 \mathrm{~Hz}$, demonstraram que, quanto maior a freqüência da EMTr, maior seria o aumento da excitabilidade cortical, mensurado por um aumento da amplitude do potencial motor evocado (MEP) e aumento na probabilidade de produzir-se MEPs em músculos alvos com EMT de pulso único, em uma intensidade que tinha sido sublimiar antes da estimulação. Por outro lado, Chen et al., em 1997, estimulando o córtex motor esquerdo de pacientes saudáveis a $0,9 \mathrm{~Hz}$ (baixa freqüência), por 15 minutos, demonstraram uma diminuição média na amplitude do MEP quando comparada ao período anterior à estimulação. Este efeito permaneceu por 15 minutos após a estimulação ter terminado. 
Pascual-Leone et al., em1998, demonstraram que quando a EMTr é aplicada sobre uma área, a excitabilidade cortical pode ser alterada e mantida, sendo dependente dos parâmetros da estimulação, principalmente, freqüência e intensidade. O local exato no sistema motor em que a alteração na excitabilidade ocorre ainda não é certo, podendo resultar em uma diminuição da eficácia das sinapses corticocorticais ou em uma alteração na excitabilidade do neurônio pós-sináptico corticoespinhal. Chen et al. sugerem que a explicação para os mecanismos ligados à manutenção da mudança na excitabilidade motora é que a EMTr pode induzir a LTP (long-term potentiation) ou LTD (long-term depression) em sinapses corticais, embora ainda não se tenha uma evidência disto. Todavia, já existem bons motivos para supor que a EMTr tem efeitos na plasticidade sináptica, e pesquisas futuras devem ajudar a elucidar os mecanismos moleculares da EMTr (Lappin e Ebmeier, 2002). Aplicada em áreas não-motoras corticais, a EMTr costuma exercer efeitos modulatórios sobre a excitabilidade cortical similares àqueles das áreas motoras corticais (Pascual-leone et al., 1998). A EMTr tem sido demonstrada como indutora de efeitos de longa duração em vários processos cognitivos, incluindo performance motora e memória. E importante notar a grande variedade interindividual destes efeitos da EMTr sobre a excitabilidade cortical. Algumas diferenças individuais basais em relação à excitabilidade cortical ou na configuração do córtex podem explicar a variabilidade nos efeitos da EMTr; entretanto, o mecanismo exato permanece incerto (Lappin e Ebmeier, 2002).

AEMTr de baixa freqüência está associada a uma diminuição da excitabilidade cortical (Chen et al., 1997). Supondo que essa diminuição da excitabilidade cortical possa ser por mecanismos de LTD, em recente estudo, Iyer et al. (2003) conduziram uma nova forma de aplicação de EMTr; é sabido que, in vitro, pode-se aumentar os efeitos de LTD com uma pré-estimulação de alta freqüência. Assim sendo, eles compararam dois grupos de voluntários saudáveis, um com uma préestimulação de $6 \mathrm{~Hz}$ (alta freqüência) antes da estimulação de $1 \mathrm{~Hz}$ (baixa freqüência) e outro com uma estimulação falsa antes da de $1 \mathrm{~Hz}$. Nos 60 minutos seguintes, a estimulação foi medindo, a cada dez segundos, o potencial motor evocado, que demonstrou um aumento significativamente maior da depressão cortical no grupo que teve a pré-estimulação de alta freqüência comparado com o grupo da pré-estimulação falsa. Os autores concluem sugerindo que este modelo deva ser testado em aplicações clínicas.

\section{Estudos envolvendo EMTr de baixa freqüência no tratamento da depressão}

O primeiro estudo com EMTr aplicada em pacientes deprimidos foi em 1993, por Höflich et al., no qual se descreveram resultados pobres em dois pacientes resistentes às drogas; porém, neste estudo foi utilizada uma estimulação de $0,3 \mathrm{~Hz}$ aplicada sobre o vértex e, por isso, ambos os córtices eram estimulados.

Os primeiros pesquisadores a utilizarem EMTr aplicada sobre um dos córtices apenas foram George et al. em 1995. Nesse estudo, eles mostraram que a estimulação de alta freqüência sobre o córtex préfrontal esquerdo melhorava os sintomas depressivos em seis pacientes resistentes à terapêutica com medicações e, o mais importante, em um desses pacientes, os efeitos clínicos da EMTr estavam associados com a normalização do hipometabolismo pré-frontal, demonstrado pelo PET.

Em 1996, Pascual-Leone et al. publicaram um importante artigo no Lancet, sendo o primeiro grupo a desenhar um estudo randomizado, controlado com placebo, em 17 pacientes, demonstrando melhora significativa das pontuações pela escala de Hamilton e inventário de Beck.

Recentemente, foi publicado um artigo que demonstra resultados semelhantes entre a EMTr de alta e baixa freqüência (Fitzgerald et al., 2003).

Em uma grande metanálise (Burt et al., 2002) que consistiu em comparações entre nove estudos abertos, 23 estudos controlados e três comparações com ECT, os autores sugerem que tanto a EMTr de alta quanto a de baixa freqüência têm efeitos antidepressivos; entretanto, a significância clínica ainda é incerta. Nos 23 estudos controlados analisados, os autores concluem que tanto a EMTr de baixa quanto a de alta freqüência têm efeitos antidepressivos estatisticamente superiores aos do grupo-controle, com estimulação falsa. Além disso, sugerem que a alta freqüência pode não ser necessária para aumentar os efeitos antidepressivos.

\section{Resultados obtidos com a aplicação de EMTr de baixa freqüência duas vezes por semana em pacientes deprimidos}

Em estudo realizado pelo nosso grupo na Universidade de Brasília (Boechat-Barros e Brasil-Neto, 2004), foram avaliados dez pacientes, sendo oito mulheres $\mathrm{e}$ dois homens, com diagnóstico de episódio depressivo maior segundo o DSM - IV (Manual Diagnóstico e Estatístico da Associação Americana de Psiquiatria) (American Psychiatric Association, 1994), considerados de difícil tratamento por seus psiquiatras clínicos, seja devido à falta de resposta, seja pela intolerância medicamentosa. Este é um estudo aberto, ou seja, não controlado com bobina inativa e sem encaminhamento aleatório, utilizando-se de série de casos.

Em cada paciente, foram aplicadas oito sessões de estimulação magnética transcraniana de baixa freqüência $-0,5 \mathrm{~Hz}-$, sendo duas por semana, com 
um intervalo mínimo de três dias entre elas. Cada sessão contava com cinco séries de vinte estímulos, com um intervalo de um minuto entre cada série, aplicados sobre o córtex pré-frontal dorsolateral direito. Em todos os pacientes, foi aplicada a escala Hamilton de 17 itens em três momentos: T-0 - antes da primeira aplicação; $\mathrm{T}-1$ - na metade do estudo; e T-2 - no final deste, com o objetivo de quantificar uma possível melhora.

A média da pontuação obtida pelos pacientes em T0, T1 e T2 foram respectivamente: 24,6, 15 e 12,8. A porcentagem média de melhora entre T0 e T2 foi de $50,45 \%$.

Para análise estatística, utilizou-se a dupla análise de variância por postos ( $x^{2}$ de Friedman), que evidenciou os resultados significativos $(p \leq 0,01)$. Foram colocados os escores da escala de Hamilton correspondentes aos momentos T0, T1 e T2 e testada a hipótese nula de que não haveria diferenças significativas entre os escores obtidos no início, meio e fim do tratamento. Com dois graus de liberdade, o $\mathrm{x}^{2}$ obtido foi superior ao $\mathrm{x}^{2}$ crítico, sendo rejeitada a hipótese nula.

Considerando as quatro semanas de tratamento, observamos um padrão considerado como resposta, isto é, melhora $\geq 50 \%$ na pontuação da escala de Hamilton em cinco pacientes. Já um padrão considerado como remissão, com pontuação menor do que oito (Roffman e Stern, 2004), nessa mesma escala, aconteceu em três pacientes.

A EMTr de baixa freqüência apresenta duas importantes vantagens em relação à de alta: segurança e custo. Em relação à segurança, não existem casos de crises convulsivas com a aplicação da EMTr de baixa freqüência, ao contrário da alta, em que existem sete casos descritos (Wasserman, 1996). Notese que, a despeito do aumento exponencial de publicações no campo da EMTr, não existem relatos publicados de convulsões desde 1996, quando foram publicadas as normas de segurança para sua utilização. Quanto ao custo, o aparelho de EMTr de baixa freqüência é bem mais barato do que o de alta, além de não ser necessário todo o aparato de suporte a uma possível crise convulsiva.

Este estudo sugere que a EMTr de baixa freqüência é uma técnica segura e pode trazer efeitos benéficos adicionais aos pacientes deprimidos que estão em uso de antidepressivos sem uma resposta clínica satisfatória. Entretanto, os resultados deste estudo necessitam de replicação, por se tratar de protocolo não-controlado, com pequeno número de observações, em um grupo de pacientes distribuídos heterogeneamente no que concerne à utilização de medicações antidepressivas.

\section{Referências bibliográficas}

American Psychiatric Association. Washington DC, USA, 1994.

Boechat-Barros, R.; Brasil-Neto J.P. - Estimulação magnética transcraniana na depressão: resultados obtidos com duas aplicações semanais. Revista Brasileira de Psiquiatria 26(2):100-2, 2004.

Burt, T.; Lisanby, S.H.; SACKeIm, H.A. - Neuropsychiatric applications of transcranial magnetic stimulation: a meta analysis. The International Journal of Neuropsychopharmacology 5:73-103, 2002.

Chen, R.R.; Classen, J.; Gerloff, C.; Celnik, P.; Wassermann, E.M.; Hallett, M.; Cohen, L.G. - Depression of motor cortex excitability by low-frequency transcranial magnetic stimulation. Neurology 48(5):1398-403, 1997.

George, M.S.; Wassermann, E.M.; Willians, W.A. - Daily repetitive transcranial magnetic stimoulation (rTMS) improves mood in depression. NeuroReport 6:1853-6, 1995.

IYeR, M.B.; SCHLePer, N.; Wassermann, E.M. - Priming stimulation enhances the depressant effect of low-frequency repetitive transcranial magnetic stimulation. $J$ Neurosci 23(34):10867-72, 2003.
Kaplan, H.; Sadock, B.; Greeb, J. - Compêndio de Psiquiatria Ciências do Comportamento e Psiquiatria Clínica. 7. ed. Porto Alegre: Artes Médicas, 1997. 1169p.

KECK, M.E. - rTMS as a treatment strategy in psychiatric disorders - neurobiological concepts. In: Paulus, W:; Tergau. F.; Nitsche, M.A.; Rothwell, J.C.; Ziemann, U.; Hallett, M. Transcranial magnetic stimulation and transcranial direct current stimulation. Proceedings of the Second International Magnetic Stimulation (TMS) and Transcranial Direct Current Stimulation (tDCS) Symposium, Göttingen, Germany, 11-14 June 2003. Amsterdam: Elsevier Science, p.100-16, 2003.

LAPPIN, J.M.; EBMEIER, K.P. - Transcranial magnetic stimulation in psychiatric disorders: does TMS affect cortical function by long-term potentialization? In: PascualLeone, A.; Davey, N.J.; Rothwell, J.; Wassermann, E. M.; PURI, B.K. Handbook of Transcranial Magnetic Stimulation. New York: Oxford University Press, 2002.

LI, X.; Nahas, Z.; Kozel, F.A.; Anderson, B.; Bohning, D.E.; Geroge, M.S. - Acute left prefrontal transcranial magnetic stimulation in depressed patients is associated with 
immediately increase activity in prefrontal cortical as well as subcortical regions. Biological Psychiatry55:88290, 2004.

Loo, C.K.; Sachdev, P.S.; HaindL, W.; Wen, W.; Mitchell, P.B.; Croker, V.M.; MALHI, G.S.- High (15 Hz) and low (1 Hz) frequency transcranial magnetic stimulation have different acute effects on regional cerebral blood flow in depressed patients. Psychological Medicine 33(6):997-1006, 2003.

Menkes, D.L.; Bodnar, P.; Ballesteros, R.A.; Swenson, M.R. - Right frontal lobe slow frequency repetitive transcranial magnetic stimulation (SFr-TMS) is an effective treatment for depression: a case-control pilot study of safety end efficacy. Journal of Neurology Neurosurgery and Psychiatry 67:113-5, 1999.

Nadeau, S.E.; McCoy, K.J.; Crucian, G.P.; Greer, R.A.; Rossı,F.; Bowers, D. et al. - Cerebral blood flow changes in depressed patients after treatment with repetitive transcranial magnetic stimulation: evidence of individual variability. Neuropsychiatry Neuropsychol Behav Neurol, 15(3):59-75, 2002.

Pascual-Leone, A.; Valls-Sole, J.; Wassermann, E.M.; Hallett, M. - Responses to rapid-rate transcranial magnetic stimulation of the human motor cortex. Brain 117 (Pt 4):847-58, 1994.

Pascual-Leone, A.; Rubio, B.; Pallardó, F.; Catalá, M.D. - Rapidrate transcranial magnetic stimulation of left dorsolateral prefrontal cortex in drug-resistant depression. Lancet 348 (9032):959, 1996.

Pascual-Leone, A.; Tormos, J.M.; Keenan, J.; Tarazona, F.; Canete, C.; CATALA, M.D. - Study and modulation of human cortical excitability with transcranial magnetic stimulation. $J$ Clin Neurophysiol 15(4):333-43, 1998.

RoffmAn, J.L.; Stern, T.A. - Diagnostic rating scales and laboratory tests. In: Stern, T.A.; Fricchione, G.L.; Cassem, N.H.; Jellick, M.S.; Rosenbaum, J.F. Handbook of General Hospital Psychiatry. Philadelphia: Elsevier, p.29-36, 2004.

Speer, A.M.; Kimbrell, T.A.; Wassermann, E,M.; D Repella, J.; Willis, M.W.; HeRsCovitch, P.; Post, R.M. - Oppositive effects of high and low frequency rTMS on regional brain activity in depressed patients. Biological Psychiatry 48:1133-41, 2000.

TenebaCK, C.C.; Nahas, Z.; SPeer, A.M.; Molloy, M.; Stalings, L.E.; SPICER, K.M. et al. - Changes in prefrontal cortex and paralimbic activity in depression following two weeks of daily left prefrontal TMS. J Neuropsychiatry Clin Neurosci 11(4):426-35, 1999.

WASSERMANN, E.M. - Risk and safety of repetitive transcranial magnetic stimulation: report and suggested guidelines from the International Workshop on the Safety of repetitive Transcranial Magnetic Stimulation. Eletroencephalography Clinical and Neurophysiology 108:1-16, 1998. 